\title{
Strong dark matter constraints on GMSB models
}

\author{
F. Staub, ${ }^{a}$ J. Niemeyer ${ }^{b}$ and W. Porod ${ }^{a}$ \\ ${ }^{a}$ Institut für Theoretische Physik und Astronomie, Universität Würzburg, \\ Am Hubland, 97074 Würzburg, Germany \\ ${ }^{b}$ Institut für Astrophysik, Universität Göttingen, \\ Friedrich-Hund-Platz 1, 37077 Göttingen, Germany \\ E-mail: florian.staub@physik.uni-wuerzburg.de, \\ niemeyer@astro.physik.uni-goettingen.de, \\ porod@physik.uni-wuerzburg.de
}

ABSTRACT: We reconsider the dark matter problem in supersymmetric models with gauge mediated supersymmetry breaking, with and without $R$-parity breaking. In these classes of models, a light gravitino forms the dark matter. Consistency with the experimental data, in particular the dark matter abundance and the small-scale power spectrum, requires additional entropy production after the decoupling of the gravitino from the thermal bath. We demonstrate that the usual mechanism via messenger number violating interactions does not work in models where the messenger belongs to $\mathrm{SU}(5)$ representations. This is mainly a consequence of two facts: (i) there are at least two different types of lightest messenger particles and (ii) the lightest messenger particle with $\mathrm{SU}(2)$ quantum numbers decays dominantly into vector bosons once messenger number is broken, a feature which has been overlooked so far. In case of $\mathrm{SO}(10)$ messenger multiplets we find scenarios which work if the SM gauge singlet component is rather light.

KEYWORDS: Supersymmetry Phenomenology

ARXIV EPRINT: 0907.0530 


\section{Contents}

1 Introduction 1

2 Models 4

3 Decay properties of messenger particles and the NLSP 5

4 Dark matter $\quad 7$

$\begin{array}{lll}5 & \text { Variations of the messenger sector } & 9\end{array}$

$\begin{array}{llr}6 & \text { Conclusions } & 10\end{array}$

\section{Introduction}

One of the most attractive features of the minimal supersymmetric standard model (MSSM) is the existence of a stable particle, usually the lightest neutralino, which successfully serves as a dark matter candidate yielding the correct amount of dark matter required by observations. Models in which the effects of supersymmetry (SUSY) breaking are communicated via the usual gauge interactions [1-9] to the "visible" have recently achieved considerable attention (see e.g. [10-15] and references therein), in particular in view of model building and understanding the mechanism of supersymmetry breaking. An attractive feature of such models is the natural explanation for the smallness of SUSY contributions to flavourchanging neutral current phenomena due to the strongly constrained SUSY spectrum.

Before discussing this model in more detail, let us briefly recall important early universe issues which are usually more or less implicitly assumed when discussing dark matter constraints within the MSSM framework:

(i) The particle content of the universe at the end of inflation is assumed to be described by the MSSM plus graviton and gravitino. This implies that the hidden sector responsible for supersymmetry breaking and/or its communication to the MSSM is essentially heavier than the reheating temperature. As a consequence, it is not produced during the reheating phase and has no impact on the later evolution of the universe.

(ii) All MSSM particles are initially in thermal equilibrium.

(iii) The gravitino may or may not be in thermal equilibrium. In the former case its number density depends strongly on the reheating temperature. 
Items (i) and (ii) allow, for example, a routine relic density calculation for thermally produced dark matter in minimal supergravity (mSUGRA) scenarios with a neutralino as the lightest supersymmetric particle (LSP) (and also in similar scenarios). Item (iii) often creates problems: in scenarios where the gravitino is the LSP, the next to lightest supersymmetric particle (NLSP) can be rather long-lived with life times up to several years. In scenarios where the gravitino is not the LSP, it itself can be similarly long-lived. In both cases one has to check that the corresponding decays do not spoil the successful predictions of big-bang nucleosynthesis (BBN).

In gauge mediated SUSY breaking (GMSB) models there are two new sectors $[6-9,16]$ :

1. the secluded sector: This is a strongly interacting sector in which SUSY is broken dynamically.

2. the messenger sector: It contains fields charged under $\mathrm{SU}(3) \times \mathrm{SU}(2) \times \mathrm{U}(1)$ gauge interactions which communicate SUSY breaking to the ordinary sparticles. Usually one assumes that they come in complete $\mathrm{SU}(5)$ representations or representations of larger groups containing $\mathrm{SU}(5)$ as subgroup, so that the success of gauge coupling unification does not get spoiled.

In these scenarios the gravitino is usually very light, in the range between a few $\mathrm{eV}$ up to $\mathrm{O}(1) \mathrm{MeV}$. The masses of the messenger particles and some of the fields in the secluded sector can be as low as $100 \mathrm{TeV}$ implying that they can act as cold dark matter if their masses are below the reheat temperature and if they are stable.

The gravitino is the lightest SUSY particle in GMSB models and all MSSM particles decay into it in a cosmologically short time. Therefore, the gravitino forms the dark matter in these models. This aspect has been extensively discussed in the literature [1722]. The abundance of thermal produced gravitinos is under assumptions consistent with the standard thermal evolution of the early universe given by

$$
\Omega_{3 / 2} h^{2}=\frac{m_{3 / 2}}{\mathrm{keV}} \frac{100}{g_{\star}} .
$$

Here $g_{\star}$ is the effective number of degrees of freedom at the time of gravitino decoupling. This implies that the gravitino forms warm dark matter (WDM) in the mass range of $O(100) \mathrm{eV}$.

However, there are stringent constraints on the contribution of WDM particles with free-streaming lengths of the order of galaxy scales or larger to the total dark matter content. More precisely, if dark matter is assumed to consist of only one particle species, its mass is limited from below by the amplitude of the small-scale power spectrum which, in turn, currently receives its tightest constraints from observations of the Lyman- $\alpha$ forest [23]. This bound has recently been increased by an order of magnitude ruling out pure WDM scenarios with particle masses below $8 \mathrm{keV}$ for non resonantly produced dark matter [24]. Gravitinos with masses up to $O(\mathrm{MeV})$ have once been in thermal equilibrium as long as the reheating temperature is above $10^{6} \mathrm{GeV}$. For those thermal relics the bound is $1.5 \mathrm{keV}$, while mixed dark matter scenarios dominated by a cold component allow a contribution 
of up to $60 \%$ by a WDM particle of any mass above $1.1 \mathrm{keV}$. There remain a number of systematic uncertainties in the interpretation of Lyman- $\alpha$ observations, most of which related to the poorly understood thermal evolution of the intergalactic medium, but the overall result is fairly robust (for a detailed discussion, see [24]), so that pure gravitino dark matter allowed by Lyman- $\alpha$ bounds would have a relic density at least 15 times higher than the measured dark matter relic density.

An additional difficulty stems from the fact that in typical GMSB models, the lightest messenger particle is stable as a result of the conservation of a messenger quantum number. Its relic density is calculable similarly to the case of a neutralino LSP and is found to scale as $\Omega_{M} h^{2} \simeq 10^{5} m_{-}^{2} /\left(10^{3} \mathrm{TeV}\right)^{2}$, where $m_{-}$is the mass of the lightest messenger particle. This overcloses the universe in most of the parameter space as discussed below. The situation becomes even worse because, as we will show below, there are usually several different types of stable messenger particles, one for each type of the corresponding SM gauge group representations.

A possible solution to both problems is additional entropy production by decays of the lightest messenger particles into standard model (SM) fermions [18-22]. The basic idea is that, in general, gravitational interactions break global symmetries and thus one expects terms like $f m_{3 / 2} \hat{\Phi}_{M} \hat{\overline{5}}$ in the superpotential [22] where $\hat{\Phi}_{M}$ is a messenger 5-plet and $\hat{\overline{5}}$ is a 5-plet containing the right down quark superfield and the left lepton superfields of the MSSM. Owing to such interactions, the lightest messenger field decays into standard model fermions. It has been claimed in the literature that it is sufficiently long lived to substantially produce entropy, diluting the gravitino abundance. This in turn would imply that heavier gravitinos with masses above $8 \mathrm{keV}$ would be viable DM candidates. However, we will show below that this statement is incorrect as only part of the possible messenger decay modes have been taken into account in the literature.

So far we have implicitly assumed that $R$-parity is conserved implying a stable LSP. There is one experimental observation which can be explained by the breaking of $R$-parity, namely neutrino masses and mixings. In the simplest model, one adds bilinear $R$-parity breaking terms to the MSSM superpotential and in this way neutrino data can be explained, see e.g. [25, 26] and references therein. Moreover, this class of models is also consistent with constraints from baryogenesis [27]. Now, what about dark matter? Neutrino physics gives a lower bound on the $R$-parity breaking parameters such that the lightest MSSM particle will decay within a small fraction of a second. However, this does not apply to a light gravitino, which eventually decays, but neutrino physics now implies that its life time is much larger than the age of universe [28-30].

The plan of this paper is as follows: in the next section we will briefly discuss the main features of the models under consideration. In section 3 we will discuss the decays of the lightest messenger pointing out the importance of decays into vector bosons, which have not been discussed so far in the literature. In section 4 we discuss the consequences for dark matter and in section 6 we conclude. 


\section{Models}

Here, we briefly recall the main features of GMSB models needed for the subsequent discussions. Further details can be found in ref. [16]. For the following discussion a minimal model is sufficient, e.g., we assume one pair of 5-plets of messenger fields coupled to one spurion superfield $\hat{S}$ in the secluded sector. The resulting superpotential can be written as

$$
W=W_{M S S M}+\hat{S} \hat{\Phi}_{M} \hat{\bar{\Phi}}_{M}+W_{s}\left(\hat{S}, \hat{Z}_{i}\right)
$$

where $W_{M S S M}$ contains the MSSM superpotential and $W_{s}$ is the superpotential of the secluded sector containing the fields $\hat{S}$ and $\hat{Z}_{i}$. The field $\hat{S}$ is one of the fields responsible for SUSY breaking and both the scalar component and the auxiliary component receive vacuum expectation values (vevs) denoted by $M$ and $F$, respectively. In the resulting effective action, $\hat{S}$ can be replaced by $M+\theta \theta F$. As a consequence of SUSY breaking, the messenger fermion receives a mass $M$ and the scalar components obtain masses $m_{-,+}=$ $M \sqrt{1 \mp F / M^{2}}$ and the corresponding eigenstates are $\phi_{-}=\left(-\Phi_{M}^{*}+\bar{\Phi}_{M}\right) / \sqrt{2}$ and $\phi_{+}=$ $\left(\Phi_{M}^{*}+\bar{\Phi}_{M}\right) / \sqrt{2}$. Strictly speaking, these formulas are valid at the GUT scale and different components get renormalized differently due to differences in the corresponding gauge couplings, resulting in a somewhat lighter $\mathrm{SU}(2)$ doublet with the same quantum numbers as the lepton doublet and a heavier $\mathrm{SU}(3)$ triplet state carrying the same quantum numbers as the right-handed $d$-quarks. However, the mass formula applies for both messenger types. Note that the requirement $\_>0$ implies $F \leq M^{2}$. Moreover, both the lightest d-type messenger scalar and the lightest doublet scalar are stable due to gauge invariance, e.g., there are no kinematically allowed decay processes from one of these two fields into the second one.

Since the messenger fields share the SM gauge interactions, the gaugino and scalar SUSY particles obtain masses at the one- and two-loop level [16], respectively:

$$
M_{i} \sim \frac{\alpha_{i}}{4 \pi} \frac{F}{M}, \quad m_{x}^{2} \sim \sum_{a} C_{a}\left(\frac{\alpha_{a}}{4 \pi}\right)^{2}\left(\frac{F}{M}\right)^{2},
$$

where the sum runs over the gauge couplings corresponding the quantum numbers of the MSSM field $x$, and $C_{a}$ is the Casimir of the corresponding representation. In order to obtain masses of the order $100 \mathrm{GeV}$ to $1 \mathrm{TeV}$, one finds that $\Lambda \equiv F / M \sim 100 \mathrm{TeV}$. The gravitino mass is related to the fundamental SUSY breaking scale

$$
m_{3 / 2}=\frac{F_{\mathrm{tot}}}{\sqrt{3} m_{P l}}
$$

where $F_{\text {tot }}=F+\sum_{i} F_{Z_{i}}$ is the sum of all $F$-terms in the secluded sector and $m_{P l}$ is the reduced Planck mass. Defining $k=F / F_{\text {tot }}$, one can write $m_{3 / 2}=F /\left(\sqrt{3} k m_{P l}\right)$.

Messenger gauge interactions and those derived from eq. (2.1) conserve messenger number so that the lightest messenger boson is stable in this minimal version of the model. In scenarios where its mass is below the reheating temperature, this leads in general to an over-closure of the universe [17]. However, on general grounds one expects that gravitational interactions either break this discrete symmetry and/or induce higher-dimensional 
operators [18-21] implying that the lightest messenger particle becomes unstable. A thorough discussion of the various possibilities and the related theoretical uncertainties can be found in ref. [22]. The general conclusion has been that in a large part of the parameter space, the lightest messenger decays after the freeze out of the gravitino and before BBN, thus allowing for a gravitino with masses of several $\mathrm{keV}$. To be specific we consider the case

$$
\delta K=f m_{3 / 2} \hat{\Phi}_{M} \hat{\overline{5}}
$$

is added to the minimal Kähler potential $K_{0}=\sum_{i} \hat{\Phi}_{i}^{\dagger} \hat{\Phi}_{i}$ where the sum runs over all superfields. The constant $f$ is usually assumed to be $O(1)$ but we will take it as a free parameter allowing arbitrary values. Using the usual invariance of the supergravity Lagrangian under Kähler transformations $K \rightarrow K+F(\hat{\Phi})+F^{*}\left(\hat{\Phi}^{*}\right), F=-\delta K$, followed by the superpotential scaling $W \rightarrow e^{-F} W[31]$ one obtains in lowest order the following additional contribution to the superpotential

$$
\delta W=f m_{3 / 2} \hat{\Phi}_{M} \hat{\overline{5}}
$$

As we discuss in some detail later, this term leads to various decays of the lightest messenger scalar.

The model(s) described so far conserve $R$-parity. As mentioned above, one can explain neutrino data by breaking $R$-parity via lepton number breaking terms. The simplest model consistent with the experimental data is obtained by adding bilinear terms to the MSSM superpotential $[25,26]$ :

$$
W_{M S S M} \rightarrow W_{M S S M}+\epsilon_{i} \hat{L}_{i} \hat{H}_{u}
$$

This way, one cannot only explain neutrino data but also predict certain decay properties of the LSP within the MSSM spectrum in terms of neutrino mixing angles [32-34]. In our case, the lightest SUSY particle is the gravitino and these predictions hold for the NLSP [30]. Eventually also the gravitino will decay but its life time is of the order of $10^{30}$ Hubble times [28-30] and, thus, the gravitino is in principle a valid DM candidate in this class of models. An interesting question, which will be addressed below, is whether the NLSP can be long lived enough so that its decay products yield additional entropy production once the gravitino has been decoupled from the thermal bath.

\section{Decay properties of messenger particles and the NLSP}

In GMSB models there are serious problems with cosmology as discussed above: (i) if only the gravitino were responsible for dark matter assuming the standard history of the universe, it would be warm dark matter with a mass of about $100 \mathrm{eV}$, which is in conflict with the measurements of the Lyman- $\alpha$ forest. (ii) If messenger particles are produced after inflation and if messenger number is conserved, one will in general obtain an exceedingly large contribution to $\Omega h^{2}$ which overcloses the universe. Both problems can, in principle, be solved by breaking messenger number, implying that the lightest messenger particles decay and give rise to sufficient additional entropy production once the gravitino is decoupled from the thermal bath, which gives a lower bound on the life time of these particles. 
Let us first consider the spectrum of the models before discussing the decay properties of the various particles. In the model with conserved messenger number and conserved $R$-parity one has $m_{+}>M>m_{-} \gg m_{S U S Y} \gg m_{3 / 2}$. Both, $f m_{3 / 2}$ in eq. (2.5) and $\epsilon_{i}$ in eq. (2.6), are small compared to $m_{S U S Y}$ implying that the induced mixing will only give small corrections to the various masses. This spectrum gives rise to decays of the following type: $\phi_{+} \rightarrow \tilde{B} \tilde{\phi}$ and $\tilde{\phi} \rightarrow \tilde{B} \phi_{-}$, where $\tilde{\phi}$ is the messenger fermion. These decays are so fast that $\phi_{+}$and $\tilde{\phi}$ will decay immediately after their decoupling from the thermal bath.

The term in eq. (2.5) induces mixings between the messenger scalars and the MSSM sfermions, in particular between messenger SU(3) triplet and the squarks and between the messenger $\mathrm{SU}(2)$ doublet and the sleptons. For example, in the model with conserved $R$-parity one obtains for the mixing matrix between the sleptons with $\mathrm{SU}(2)$ doublet the following mass matrix in the basis $\left(\tilde{L}_{i}, \phi_{-}\right)$where $\tilde{L}$ are the left-sleptons:

$$
\left(\begin{array}{cccc}
M_{L, 11}^{2}+D+f^{2} m_{3 / 2}^{2} & 0 & 0 & \frac{1}{\sqrt{2}} f m_{3 / 2} M \\
0 & M_{L, 22}^{2}+D+f^{2} m_{3 / 2}^{2} & 0 & \frac{1}{\sqrt{2}} f m_{3 / 2} M \\
0 & 0 & M_{L, 33}^{2}+D+f^{2} m_{3 / 2}^{2} & \frac{1}{\sqrt{2}} f m_{3 / 2} M \\
\frac{1}{\sqrt{2}} f m_{3 / 2} M & \frac{1}{\sqrt{2}} f m_{3 / 2} M & \frac{1}{\sqrt{2}} f m_{3 / 2} M & M^{2}-F+D^{\prime}+\frac{3}{2} f^{2} m_{3 / 2}^{2}
\end{array}\right)
$$

where $D$ and $D^{\prime}$ denote $D$-terms occurring after electro-weak symmetry breaking with $D=O\left(m_{Z}^{2}\right)$ and $D^{\prime} \simeq 0$. The latter is due to the fact $D^{\prime}$ is propotional to $\cos 2 \theta$ where $\theta$ is the mixing angle between $\phi_{-}$and $\phi_{+}$which turns out to be maximal: $\theta \simeq 1 / \sqrt{2}$. For simplicity, we have assumed that the couplings of the messenger superfield is generation independent, e.g. $f_{1}=f_{2}=f_{3}=f$. Relaxing this assumption does not change any of our conclusions. The induced mixing between the sneutrinos and the neutral messenger scalar is of the order

$$
\delta \simeq \frac{f m_{3 / 2} M}{\sqrt{2}\left(M^{2}-F\right)} \simeq \frac{f m_{3 / 2} M}{\sqrt{2} m_{-}^{2}}
$$

In the literature $[19,20,22]$ it is assumed that $F \ll M^{2}$ and the lightest messenger decays predominantly into a neutralino and a SM fermion. The generic decay width is calculated to

$$
\Gamma \simeq \frac{g^{2}}{16 \pi} \delta^{2} m_{-} .
$$

This would imply that the lightest messenger scalars decay after the freeze out of the gravitinos and before BBN. Furthermore, the dilution for a natural choice of parameters, e.g. $f=O(1)$ could lead to a relic density of the gravitinos of $\Omega h^{2} \simeq 0.1$.

This conclusion would be correct in the absence of an event which has not been taken into account so far: electroweak symmetry breaking which opens new decay channels, namely into vector-bosons. It has been shown [35, 36] that decays into $W$ - and $Z$-bosons can dominate the decays of supersymmetric scalar particles if there is sufficient phase space. It turns out that in our scenario, the decay into $Z$ bosons are suppressed via an extended GIM mechanism. However, this is not the case for decays into $W$-bosons and one gets for the corresponding widths

$$
\Gamma \simeq \frac{g^{2}}{16 \pi} \frac{m_{-}^{2}}{m_{W}^{2}} \delta^{2} m_{-} .
$$


The additional factor $\frac{m_{-}^{2}}{m_{W}^{2}}$ implies that this decay mode dominates once the lightest messenger scalar has a mass of a few $\mathrm{TeV}$ and its life time gets reduced by the inverse factor. Therefore, it is of vital importance to know how small $m_{-}$can be as this gives an upper bound on the life-time. At tree level one might argue that $\sqrt{M^{2}-F} \simeq m_{W}$ but it turns out that the one-loop corrections dominate the mass in this case:

$$
\delta m^{2}=\sum_{i} \frac{1}{4 \pi^{2}} C_{i}^{2} g_{i}^{2} M^{2} .
$$

The sum is taken over contributing gauge couplings and $C_{i}^{2}$ is the corresponding Dynkin index. This can be easily understood by noting that at the scale $M$, the messenger fermions decouple from the spectrum and, thus, supersymmetry is broken as the number of bosonic degrees of freedom does not match the number of fermionic degrees of freedom leading to this large correction. Additionally the masses are increased by the RGE running from the SUSY breaking scale to the low scale by a factor $\left(1-\frac{C_{i}^{2} g_{i}^{2}\left(M_{Z}\right)}{8 \pi^{2}} \ln \left(\frac{M^{2}}{M_{Z}^{2}}\right)\right)^{-1}$.

We made a full analysis of the possible decays of the lightest messenger using the one loop corrections for the messenger masses. The SUSY parameters were calculated with SPheno [37] and the $\mu / B_{\mu}$ parameters were fixed by solving the tadpole equations. All vertices and mass matrices of the model were derived with SARAH [38]. The result is that taking the one-loop corrections to the mass of $\phi_{-}$implies that the final state $W \tilde{l}_{L}$ dominates the decays of $\phi_{-}$such that all other decay modes have a branching ratio of at most $10^{-6}$. This, in turn, implies a total width in the order of $\mathrm{meV}$ as can be seen in figure 1 for the two limiting cases $F \ll M^{2}$ and $F \simeq M^{2}$ with dramatic consequences for the dark matter considerations as discussed in the next section. As can be seen, due to the inclusion of $W^{-}$final state the width increases such, that the decay temperature of $\phi_{-}$is above the freeze out temperature of the gravitino.

In models with broken $R$-parity one might wonder if the NLSP decays might yield additional entropy production after the freeze-out of the gravitino. However, in this model a lower bound on the corresponding couplings exist due to requirement of correctly explaining neutrino physics [32-34]. In case of a neutralino NLSP there are regions in parameter space where it is long-lived enough to see a displaced vertex at LHC [39] but, nevertheless, it is too short-lived to be of importance for cosmological considerations. A stau NLSP decays even faster and, thus, does not play a role either.

\section{Dark matter}

Owing to the mixing term in eq. (2.5), the lightest messenger particle ceases to be stable but decays into SM and SUSY particles. The ratio of the entropy before and after the decay of the messengers is estimated by [40]

$$
\frac{s_{\text {after }}}{s_{\text {before }}} \simeq \frac{4}{3} \frac{Y m}{T_{D}}
$$

and leads to a dilution of the relic density of particles already frozen out. The question is now whether one can get sufficient dilution after taking into account the additional decay 

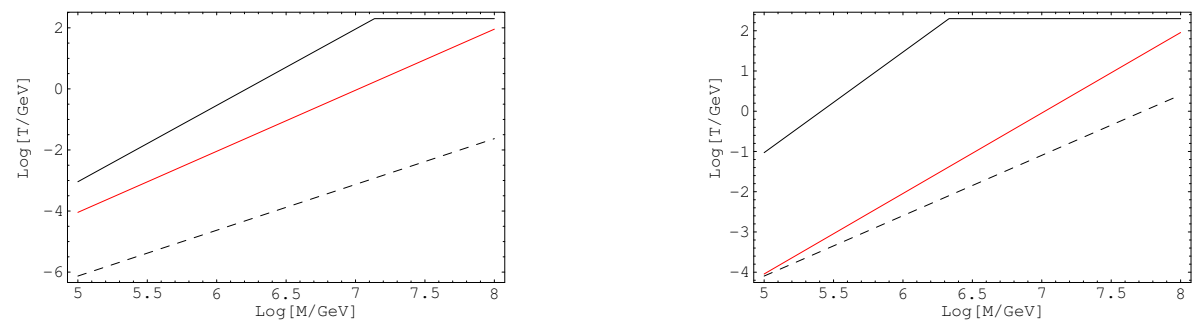

Figure 1. Comparison of the the decay temperature of the messenger to the freeze out temperature of the gravitino (red line). The black, solid line shows the temperature including the decay in $W^{-}$, the dashed line the temperature without this channel. Left plot for $M^{2} \gg F$ and right plot for $M^{2} \cong F$, both for $k=1, f=1$.

mode $\phi_{-} \rightarrow W \tilde{l}_{L}$. The freeze-out temperature of the gravitino is given by [16]

$$
T_{3 / 2}=0.62 \frac{m_{3 / 2}^{2} M_{P \sqrt{g_{\star}}}}{\alpha_{S} m_{\tilde{g}}^{2}} .
$$

The gluino mass is given by $m_{\tilde{g}} \simeq \frac{\alpha_{s}}{4 \pi} \Lambda$ [16] in GMSB. As we only need a rough estimate of this temperature, we use eq. (2.3) and set all parameters of $O(1)$ to 1 . As a result, we get $T_{3 / 2}$ as a function of the messenger mass $M: T_{3 / 2} \simeq 10^{-13} \frac{M^{2}}{k^{2} \mathrm{GeV}}$. Comparing this to the decay temperature of the scalar messengers in figure 1, one sees that the $\mathrm{SU}(2)$ doublet messenger always decays before the freeze-out of the gravitinos has taken place. This effect is independent of the choice of the free parameters and of the fact that we have considered only one pair of messengers thus far. As mentioned above, also the lightest SU(3) messenger is stable in the limit of conserved messenger number and once this number is broken it decays according to eq. (3.3) dominantly into a $d$-quark and a gluino with the correct life time. However, although the life time is of the required order of magnitude, the number density is too small to provide sufficient entropy. This can be seen in figure 2, where the necessary relic density for sufficient dilution is compared with the obtainable density. For tiny ratios of $\frac{\Lambda}{M}$, the annihilation in gravitinos becomes dominant, which is reflected in the strong bending of the dashed lines in the plot to the right of figure 2, and inhibits a bigger dilution. Since the interactions of the Goldstino are proportional to $m_{3 / 2}^{-1}$ the situation gets worse for larger values of $k$. The sharp edges of the blue lines in the left plot arise because the degrees of freedom change, when the decay temperatur crosses a mass threshold, as can be seen from eq. (1.1). Note that in figure 2 we have covered for completeness a much larger part of the parameter space than the one accessible at LHC: mainly the parameter space shown in the right figure has a sufficiently light spectrum whereas in most the left plot the spectrum is too heavy for detection at LHC. Moreover, in the green area one finds a gravitino mass larger then $1 \mathrm{MeV}$ and, thus, one can avoid the problem of over-closing the universe if the reheat temperature is sufficiently low [45].

Up to now we have assumed that $f$ is an $O(1)$ parameter. As mentioned above it results from a non-minimal Kähler potential and naturally is $O(1)$ [22]. As the widths of the lightest messengers are proportional to $f^{2}$ one might take smaller values to increase the life-time of the SU(2) messenger. However, this does not work in practice, because the 

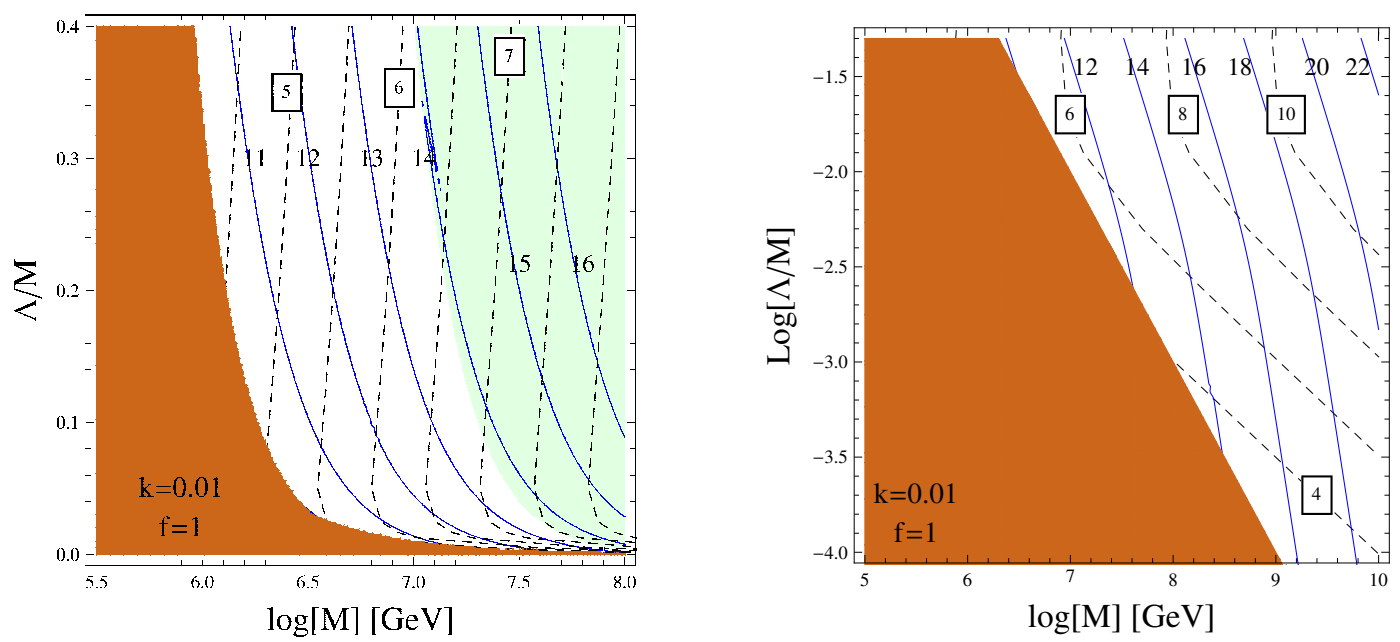

Figure 2. Comparison between the calculated relic density for the lightest strongly interacting messenger scalar (black dashed line) and needed relic density for a sufficient dilution of the gravitinos (blue solid line). The labels show $\log \Omega$. The right plot is a zoom of the area with $\Lambda / M$ below 0.1 of the left plot. The brown area is excluded by Lyman- $\alpha$ forest observations and the condition $\Lambda>10^{5} \mathrm{GeV}$ because of LEP bounds for SUSY masses. In the green area one gets $m_{3 / 2} \geq 1 \mathrm{MeV}$.

life time of the $\mathrm{SU}(3)$ messengers also increase and once $f$ is of $O\left(10^{-2}\right)$ this destroys the successful BBN predictions: they decay at temperature in the low $\mathrm{keV}$ range and below. At this time they dominate the energy density of the universe implying that the energy injection due to their decays would dissociate BBN products to a very high extent. In addition, the reheating temperature of this decay is related to the decay temperature and the dilution factor by $T_{R H}^{\mathrm{SU}(3)}=T_{D}^{\mathrm{SU}(3)} \sqrt[3]{\Delta^{\mathrm{SU}(3)}}$. For the calculation of the dilution factor we used MicrOmegas [41]. This reheating is too low to restart BBN again, especially since the number density of the $\mathrm{SU}(3)$ messengers gets also diluted by the decay of the $\mathrm{SU}(2)$ messengers. To obtain a rough impression of the reheating temperature, we can approximate $Y_{\mathrm{SU}(3)} \simeq Y_{\mathrm{SU}(2)}$ and $M_{\mathrm{SU}(3)} \simeq M_{\mathrm{SU}(2)}$ leading to

$$
T_{R H}^{\mathrm{SU}(3)}=T_{R H}^{\mathrm{SU}(3)}\left(\frac{Y_{\mathrm{SU}(3)}}{Y_{\mathrm{SU}(2)}} \frac{M_{\mathrm{SU}(3)}}{M_{\mathrm{SU}(2)}} \frac{T_{D}^{\mathrm{SU}(2)}}{T_{D}^{\mathrm{SU}(3)}}\right)^{\frac{1}{3}} \simeq T_{D}^{\mathrm{SU}(3)}\left(\frac{g}{g_{3}} \frac{M}{M_{W}}\right)^{\frac{1}{3}}
$$

i.e. the reheating temperature remains in the $\mathrm{keV}$ range.

Therefore, it is also not possible to adjust the parameters in a way that a combined dilution of both decays leads to a correct Gravitino abundance and circumvents BBN bounds, because the ratio of the yields and of the decay temperatures is fixed by the gauge couplings, the messenger mass $M$ and the $W$ mass.

\section{$5 \quad$ Variations of the messenger sector}

So far, we have only considered a minimal GMSB scenario with one messenger pair transforming as 5 and $\overline{5}$ under $\mathrm{SU}(5)$. We have seen, that the $\mathrm{SU}(2)$ messengers could in principle produce the required additional entropy if we fine-tune their decay temperature 
to the minimal value allowed by $\mathrm{BBN}$ by reducing the parameter $f$. But the $\mathrm{SU}(3)$ messengers decay in this scenario much later, and this scenario is therefore ruled out by BBN. This result is independent of the concrete mixing term, e.g., it does not change if instead one takes higher-dimensional operators inducing the mixing of the messenger particles with the MSSM particles. We have checked that for all cases proposed in [22], which contains an exhaustive list of possibilities, the same conclusions hold. The picture changes a little bit if the messenger multiplet contains $\mathrm{U}(1)$ charged particles which are $\mathrm{SU}(3) \times \mathrm{SU}(2)$ singlets, i.e. $\tilde{e}_{R}$-like messengers. They have a relic density comparable to $\tilde{v}_{L}$-like messengers, but their decay width is not enhanced by decays into vector-bosons. Therefore, these messengers can have the smallest decay width of all messengers. However, in out numerical studies we have only found tiny isolated regions in parameter space rendering this possibility highly unattractive as this requires quite some fine-tuning of the parameters.

The last possibility is to consider a messenger sector containing singlets under all gauge groups $\left(\tilde{v}_{R}\right.$-like) as it appears for example in the 16-plet of $\mathrm{SO}(10)$. This was already analyzed in [42] where it has been shown that this scenario works in principle. Their analysis was based on annihilation due to loop vertices, which are dominant if the spurion mass is of the order of the messenger mass and $M \simeq 10^{6}$. We reconsidered this scenario for the case that all particles of the hidden sector are heavier than the messengers. As a consequence, the dominant interaction of the messengers is always due to the Goldstino component of the gravitino. Their relic density and their decay width depend only on this interaction and, thus, the decay in MSSM particles is given by

$$
\Gamma=\frac{1}{16 \pi}\left(\frac{m_{-}^{2}}{m_{3 / 2} m_{P l}}\right)^{2} \delta^{2} m_{-} .
$$

The result of our numerical studies is that for the case $M^{2} \gg F$ and $f=1$ this scenario works for very small values of $k\left(O\left(10^{-5}\right)\right)$ and messenger masses of $O\left(10^{7}\right)$, as can be seen in figure 3 on the left. For larger values of $k$ or $M$, the annihilation is too effective and the additional entropy production too small. This can be partly compensated by reducing $f$ as the dilution behaves like $\Delta \sim k^{-4} f^{-1}$ and we reach the BBN bound very fast. However, if we assume $\Lambda \simeq M$, one finds solutions for larger $k$ as can be seen figure 3 right. The reason is that the annihilation in Goldstinos, a t-channel interaction, is suppressed by the large mass splitting. The messengers are in the PeV-range and the gravitino mass is about $10 \mathrm{keV}$, i.e. it is still warm dark matter but not in conflict with the Lyman- $\alpha$ observations. Note that in contrast to charged messenger particles a $\tilde{v}_{R}$ messenger does not receive large one-loop corrections to its mass due to gauge interactions. Note that as in figure 2 we have covered in figure 3 for completeness a much larger part of the parameter space than accessible at LHC.

\section{Conclusions}

We have reconsidered the question of dark matter in gauge mediated SUSY breaking. Assuming that the reheat temperature is large enough so that the gravitino gets into thermal equilibrium, we find that in the simplest models it is not possible to obtain the 

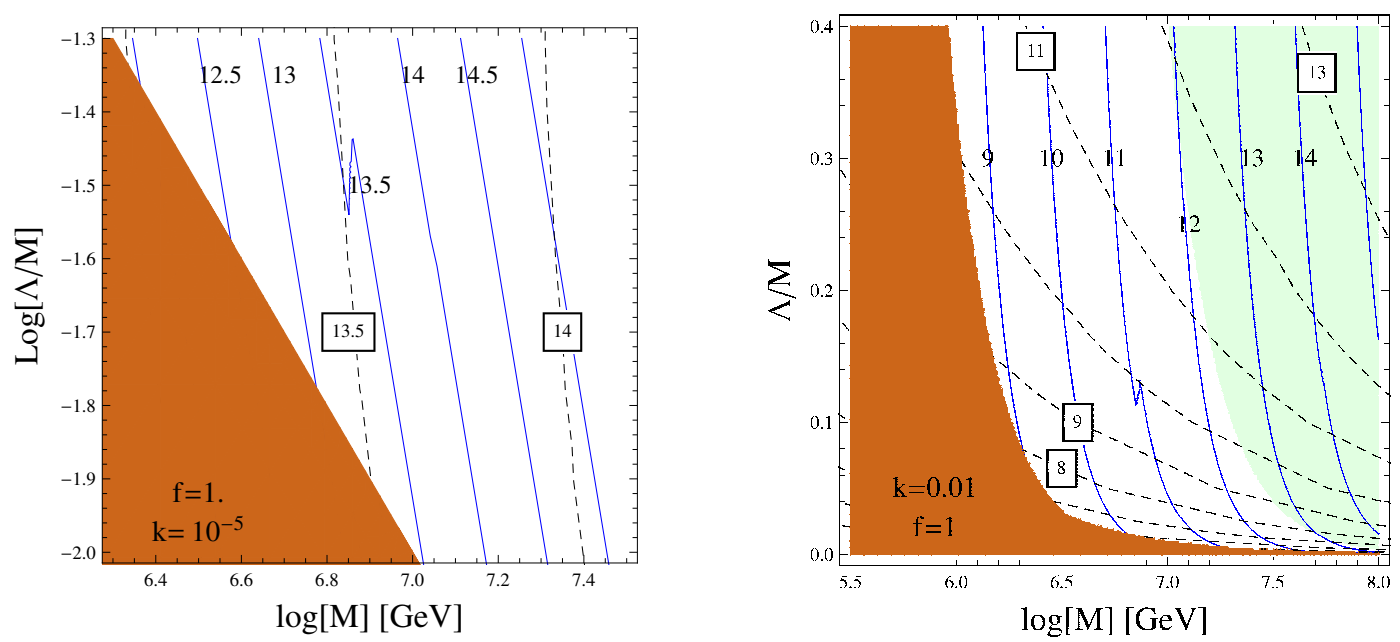

Figure 3. Comparison between the calculated relic density for gauge singlet Messenger (black dashed line) and needed relic denisty for sufficient delution (blue solid line). The labels show $\log \Omega$. Left plot for a ratio $r$ smaller 0.01 and $k=10^{5}$, right plot for larger $r$ and $k$. The brown area is excluded by Lyman- $\alpha$-forest observation and the condition $\Lambda>10^{5} \mathrm{GeV}$. In the green area one gets $m_{3 / 2} \geq 1 \mathrm{MeV}$.

correct amount of dark matter once all contraints are taken into account: observation of the Lyman- $\alpha$ forest implies that a gravitino with a mass above about $8 \mathrm{keV}$ is needed which yields too much dark matter if only the standard history of the universe is considered. The mechanisms to produce additional entropy via messenger number violating terms proposed so far do not work in scenarios where the messenger come in complete SU(5) representations as a consequence of two facts which have been overlooked so far: (i) the decay of the messenger particle has to occur after electroweak phase transistion and thus decays into $W$-bosons have to be considered and (ii) for each represenation of the SM gauge group one has a lightest messenger scalar which would be stable if messenger number were a conserved quantum number. Roughly one finds two main possiblities: the $\mathrm{SU}(2)$ messengers decay too fast and the $\mathrm{SU}(3)$ messengers do not produdce sufficient entropy to dilute the gravitinos, or the $\mathrm{SU}(2)$ messengers decay sufficiently late and the $\mathrm{SU}(3)$ messengers destroy the predictions for BBN. However, by sufficiently lowering the reheat temperature one can avoid this problem once the gravitino mass is in the range of $1 \mathrm{MeV}$ and above.

Although we have mainly focused on terms of the form $\mathrm{fm}_{3 / 2} \hat{\Phi} \hat{\overline{5}}_{M}$ in detail, one can easily extend this discussion to other scenarios. We have checked that for all cases proposed in ref. [22] which contains an exhaustive list of possibilities, the same conclustions hold but for a tiny region in parameter space where a $\tilde{e}_{R}$ like messenger is the lightest messenger particle. Moreover, we have also seen that scenarios with a SM gauge singlet lightest messenger, occuring for example in 16-plet of $\mathrm{SO}(10)$ models, are still viable.

In this study, we have assumed that the gravitino constitutes all of the dark matter. If one relaxes this assumption, one has to invoke scenarios where the gravitino has a mass below $60 \mathrm{eV}$ (see (1.1)) if at most $60 \%$ of the dark matter is allowed to be warm dark matter [24] where this limit has been found in case of a $5 \mathrm{KeV}$ gravitino. Of course the mass gets further 
constrained if the allowed warm dark matter contribution has to be smaller. Taking into account the existing collider constraints on chargino, slepton and Higgs masses [43], we get a lower bound of about $3 \mathrm{eV}$ on the gravitino mass. In the mass range between 3 and $60 \mathrm{eV}$, the NLSP life time is such that in principle a displaced vertex can be measured at the LHC which scales roughly like $m_{3 / 2}^{2}$. Thus, one can get information on the gravitino mass once the properties of the decaying particle are known. This in turn would be a clear indication that the gravitino cannot form all of the observed dark matter. In case of a gravitino with mass of $O(1) \mathrm{eV}$, one might even find imprints in the reconstructed CMB lensing potential [44].

\section{Acknowledgments}

This work is supported by the DFG Graduiertenkolleg GRK-1147 and by the DAAD, project number $\mathrm{D} / 07 / 13468$.

Open Access. This article is distributed under the terms of the Creative Commons Attribution Noncommercial License which permits any noncommercial use, distribution, and reproduction in any medium, provided the original author(s) and source are credited.

\section{References}

[1] M. Dine and W. Fischler, A phenomenological model of particle physics based on supersymmetry, Phys. Lett. B 110 (1982) 227 [SPIRES].

[2] M. Dine, W. Fischler and M. Srednicki, Supersymmetric technicolor, Nucl. Phys. B 189 (1981) 575 [SPIRES].

[3] S. Dimopoulos and S. Raby, Supercolor, Nucl. Phys. B 192 (1981) 353 [SPIRES].

[4] C.R. Nappi and B.A. Ovrut, Supersymmetric extension of the $\mathrm{SU}(3) \times \mathrm{U}(2) \times \mathrm{U}(1)$ model, Phys. Lett. B 113 (1982) 175 [SPIRES].

[5] L. Álvarez-Gaumé, M. Claudson and M.B. Wise, Low-energy supersymmetry, Nucl. Phys. B 207 (1982) 96 [SPIRES].

[6] M. Dine and A.E. Nelson, Dynamical supersymmetry breaking at low-energies, Phys. Rev. D 48 (1993) 1277 [hep-ph/9303230] [SPIRES].

[7] M. Dine, R.G. Leigh and A. Kagan, Supersymmetry and the Nelson-Barr mechanism, Phys. Rev. D 48 (1993) 2214 [hep-ph/9303296] [SPIRES].

[8] M. Dine, A.E. Nelson and Y. Shirman, Low-energy dynamical supersymmetry breaking simplified, Phys. Rev. D 51 (1995) 1362 [hep-ph/9408384] [SPIRES].

[9] M. Dine, A.E. Nelson, Y. Nir and Y. Shirman, New tools for low-energy dynamical supersymmetry breaking, Phys. Rev. D 53 (1996) 2658 [hep-ph/9507378] [SPIRES].

[10] K.A. Intriligator, N. Seiberg and D. Shih, Dynamical SUSY breaking in meta-stable vacua, JHEP 04 (2006) 021 [hep-th/0602239] [SPIRES].

[11] H. Murayama and Y. Nomura, Gauge mediation simplified, Phys. Rev. Lett. 98 (2007) 151803 [hep-ph/0612186] [SPIRES]. 
[12] H. Murayama and Y. Nomura, Simple scheme for gauge mediation, Phys. Rev. D 75 (2007) 095011 [hep-ph/0701231] [SPIRES].

[13] L.M. Carpenter, M. Dine, G. Festuccia and J.D. Mason, Implementing general gauge mediation, Phys. Rev. D 79 (2009) 035002 [arXiv:0805.2944] [SPIRES].

[14] K.R. Dienes and B. Thomas, Building a nest at tree level: classical metastability and non-trivial vacuum structure in supersymmetric field theories, Phys. Rev. D 78 (2008) 106011 [arXiv:0806.3364] [SPIRES].

[15] Z. Komargodski and N. Seiberg, $\mu$ and general gauge mediation, JHEP 03 (2009) 072 [arXiv: 0812.3900] [SPIRES].

[16] G.F. Giudice and R. Rattazzi, Theories with gauge-mediated supersymmetry breaking, Phys. Rept. 322 (1999) 419 [hep-ph/9801271] [SPIRES].

[17] S. Dimopoulos, G.F. Giudice and A. Pomarol, Dark matter in theories of gauge-mediated supersymmetry breaking, Phys. Lett. B 389 (1996) 37 [hep-ph/9607225] [SPIRES].

[18] T. Han and R. Hempfling, Messenger sneutrinos as cold dark matter, Phys. Lett. B 415 (1997) 161 [hep-ph/9708264] [SPIRES].

[19] E.A. Baltz and H. Murayama, Gravitino warm dark matter with entropy production, JHEP 05 (2003) 067 [astro-ph/0108172] [SPIRES].

[20] M. Fujii and T. Yanagida, Natural gravitino dark matter and thermal leptogenesis in gauge-mediated supersymmetry-breaking models, Phys. Lett. B 549 (2002) 273 [hep-ph/0208191] [SPIRES].

[21] M. Fujii and T. Yanagida, Baryogenesis and gravitino dark matter in gauge-mediated supersymmetry breaking models, Phys. Rev. D 66 (2002) 123515 [hep-ph/0207339] [SPIRES].

[22] K. Jedamzik, M. Lemoine and G. Moultaka, Gravitino dark matter in gauge mediated supersymmetry breaking, Phys. Rev. D 73 (2006) 043514 [hep-ph/0506129] [SPIRES].

[23] M. Viel, J. Lesgourgues, M.G. Haehnelt, S. Matarrese and A. Riotto, Constraining warm dark matter candidates including sterile neutrinos and light gravitinos with WMAP and the Lyman- alpha forest, Phys. Rev. D 71 (2005) 063534 [astro-ph/0501562] [SPIRES].

[24] A. Boyarsky, J. Lesgourgues, O. Ruchayskiy and M. Viel, Lyman- $\alpha$ constraints on warm and on warm-plus-cold dark matter models, JCAP 05 (2009) 012 [arXiv:0812.0010] [SPIRES].

[25] J.C. Romao, M.A. Diaz, M. Hirsch, W. Porod and J.W.F. Valle, A supersymmetric solution to the solar and atmospheric neutrino problems, Phys. Rev. D 61 (2000) 071703 [hep-ph/9907499] [SPIRES].

[26] M. Hirsch, M.A. Diaz, W. Porod, J.C. Romao and J.W.F. Valle, Neutrino masses and mixings from supersymmetry with bilinear R-parity violation: A theory for solar and atmospheric neutrino oscillations, Phys. Rev. D 62 (2000) 113008 [Erratum ibid. D 65 (2002) 119901] [hep-ph/0004115] [SPIRES].

[27] A.G. Akeroyd, E.J. Chun, M.A. Diaz and D.-W. Jung, Neutrino masses, baryogenesis and bilinear R-parity violation, Phys. Lett. B 582 (2004) 64 [hep-ph/0307385] [SPIRES].

[28] S. Borgani, A. Masiero and M. Yamaguchi, Light gravitinos as mixed dark matter, Phys. Lett. B 386 (1996) 189 [hep-ph/9605222] [SPIRES].

[29] F. Takayama and M. Yamaguchi, Gravitino dark matter without R-parity, Phys. Lett. B 485 (2000) 388 [hep-ph/0005214] [SPIRES]. 
[30] M. Hirsch, W. Porod and D. Restrepo, Collider signals of gravitino dark matter in bilinearly broken R-parity, JHEP 03 (2005) 062 [hep-ph/0503059] [SPIRES].

[31] J. Wess and J. Bagger, Supersymmetry and supergravity, Princeton University Press, Princeton U.S.A. (1992) pg. 259 [SPIRES].

[32] W. Porod, M. Hirsch, J. Romao and J.W.F. Valle, Testing neutrino mixing at future collider experiments, Phys. Rev. D 63 (2001) 115004 [hep-ph/0011248] [SPIRES].

[33] M. Hirsch, W. Porod, J.C. Romao and J.W.F. Valle, Probing neutrino properties with charged scalar lepton decays, Phys. Rev. D 66 (2002) 095006 [hep-ph/0207334] [SPIRES].

[34] M. Hirsch and W. Porod, Neutrino properties and the decay of the lightest supersymmetric particle, Phys. Rev. D 68 (2003) 115007 [hep-ph/0307364] [SPIRES].

[35] A. Bartl et al., Bosonic decays of stop 2 and sbottom 2 , Phys. Lett. B 435 (1998) 118 [hep-ph/9804265] [SPIRES].

[36] A. Bartl et al., Impact of bosonic decays on the search for stau ${ }_{2}$ and sneutrino, Phys. Lett. B 460 (1999) 157 [hep-ph/9904417] [SPIRES].

[37] W. Porod, SPheno, a program for calculating supersymmetric spectra, SUSY particle decays and SUSY particle production at $e^{+} e^{-}$colliders, Comput. Phys. Commun. 153 (2003) 275 [hep-ph/0301101] [SPIRES].

[38] F. Staub, SARAH, arXiv:0806.0538 [SPIRES].

[39] F. de Campos et al., Probing bilinear R-parity violating supergravity at the LHC, JHEP 05 (2008) 048 [arXiv:0712.2156] [SPIRES].

[40] E.W. Kolb and M.S. Turner, The early universe, Front. Phys. 69 (1990) 1 [SPIRES].

[41] G. Bélanger, F. Boudjema, A. Pukhov and A. Semenov, MicrOMEGAs2.0: A program to calculate the relic density of dark matter in a generic model, Comput. Phys. Commun. 176 (2007) 367 [hep-ph/0607059] [SPIRES].

[42] M. Lemoine, G. Moultaka and K. Jedamzik, Natural gravitino dark matter in $\mathrm{SO}(10)$ gauge mediated supersymmetry breaking, Phys. Lett. B 645 (2007) 222 [hep-ph/0504021] [SPIRES].

[43] Particle Data Group collaboration, C. Amsler et al., Review of particle physics, Phys. Lett. B 667 (2008) 1 [SPIRES].

[44] K. Ichikawa, M. Kawasaki, K. Nakayama, T. Sekiguchi and T. Takahashi, Constraining light gravitino mass from cosmic microwave background, JCAP 08 (2009) 013 [arXiv:0905.2237] [SPIRES].

[45] F.D. Steffen, Dark matter candidates - axions, neutralinos, gravitinos, and axinos, Eur. Phys. J. C 59 (2009) 557 [arXiv:0811.3347] [SPIRES]. 